\title{
Investigation of Nanocrystalline Materials by Perturbed Angular Correlation and Supplementing Experimental Techniques
}

\author{
H. WOLF \\ Technische Physik, Universität des Saarlandes \\ 66123 Saarbrücken, Germany
}

\begin{abstract}
Nanocrystalline materials were investigated by perturbed $\gamma \gamma$-angular correlation using the radioactive probe ${ }^{111} \mathrm{In}$. For identifying local structures observed by perturbed $\gamma \gamma$-angular correlation various supplementing experimental techniques, like X-ray diffraction, transmission electron microscopy, absorption spectroscopy, and photoluminescence spectroscopy, were applied. In nanocrystalline TiAl alloys different crystallographic structures were observed depending on the conditions of sample treatment. Nanocrystalline $\mathrm{Ni}$ samples show a slightly modified local magnetic field in regions adjacent to the grain boundaries. In addition, ordered grain boundary structures were observed. $\mathrm{Ni}$ precipitates were identified in nanocrystalline $\mathrm{NiCu}$ alloys by perturbed $\gamma \gamma$-angular correlation and preparation conditions avoiding these precipitates were found. For nanocrystalline $\mathrm{ZnO}$ preparation conditions were found yielding In-doped particles of good crystalline quality.

PACS numbers: 68.35.Ct, 75.75.+a, 78.67.Bf, 81.07.Bc, 82.45.Qr
\end{abstract}

\section{Introduction}

The properties of nanocrystalline materials are essentially determined by grain size and properties of the grain boundaries of the respective material [1]. Using perturbed $\gamma \gamma$-angular correlation (PAC) valuable information about local crystalline structures of the grains or the grain boundaries is obtained. PAC detects the hyperfine interaction of the nucleus of a suitable radioactive probe atom, here ${ }^{111} \mathrm{In}$, decaying to ${ }^{111} \mathrm{Cd}$, with an electric field gradient (EFG) or magnetic field. The hyperfine interaction is detected by recording the $\gamma-\gamma$ cascade, which is emitted after the radioactive decay of the probe nucleus, in delayed coincidence. The hyperfine interaction causes a modulation of the exponential decay function of the intermediate level of the $\gamma-\gamma$ cascade. The modulation function $R(t)$ is governed by a frequency triplet in case of an electric quadrupole interaction, 


$$
R(T)=A_{2}\left(s_{0}+\sum_{n=1}^{3} s_{n} \cos \left(\omega_{n} t\right)\right),
$$

where $A_{2}$ is the anisotropy coefficient of the $\gamma-\gamma$ cascade. For the frequencies the relation $\omega_{1}+\omega_{2}=\omega_{3}$ holds, the magnitude of the frequency $\omega_{1}$ is determined by the strength of the local EFG, which usually is expressed by the quadrupole coupling constant $\nu_{Q} \sim \omega_{1} \sim V_{z z}$. The ratio $\omega_{2} / \omega_{1}$ is determined by the asymmetry parameter $\eta=\left(V_{x x}-V_{y y}\right) / V_{z z}$ of the EFG tensor. In case of a magnetic dipole interaction the modulation is governed by a frequency doublet,

$$
R(T)=A_{2}\left(s_{0}+\sum_{n=1}^{2} s_{n} \cos \left(n \omega_{\mathrm{L}} t\right)\right)
$$

where the Larmor frequency $\omega_{\mathrm{L}}$ is proportional to the strength of the local magnetic field. If several, non-equivalent lattice sites are occupied by the probe atoms, the modulation function is split into different fractions,

$$
R(t)=\sum_{i} f_{i} R_{i}(t) \quad \text { with } \quad \sum_{i} f_{i}=1
$$

each characterized by their own frequency triplet or doublet, working like a fingerprint of the corresponding local structure. More details about PAC and its applications can be found in [2-4].

\section{Results and discussion}

For PAC investigations the samples have to be doped with radioactive ${ }^{111} \mathrm{In}$ atoms. Depending on the method of preparation the probe atoms are distributed evenly over the sample, or, in special cases, preferably placed into the grain boundaries. By annealing nanocrystalline materials, new crystalline phases might be formed, which can be detected by PAC measurements. For an assignment of the respective $\mathrm{PAC}$ signal to a distinct local lattice structure, in general, investigations by supplementing experimental techniques are necessary. In the following, some examples will be discussed showing the versatility of information obtainable by PAC investigations.

\subsection{Phase formation in TiAl alloys}

Nanocrystalline TiAl alloys were prepared by ball milling using a standard Spex 8000 ball mill. For this purpose, powders of Ti (purity 99.98\%) and of $\mathrm{Al}(99.99 \%)$ with particle sizes smaller than $150 \mu \mathrm{m}$ were mixed and filled into hardened steel vials under Ar atmosphere. The milling times ranged between $2 \mathrm{~h}$ and $52 \mathrm{~h}$. Subsequently, ${ }^{111} \mathrm{In}$ atoms were diffused into the sample at $690 \mathrm{~K}$ in an evacuated quartz ampoule. PAC spectra were also taken after ${ }^{111}$ In diffusion under additional annealing at various temperatures up to $1060 \mathrm{~K}$. PAC data from samples of composition $\mathrm{Ti}_{0.5} \mathrm{Al}_{0.5}$ are shown in Fig. 1 for a milling time of $4 \mathrm{~h}$ and in Fig. 2 for a milling time of $13 \mathrm{~h}$. After diffusion of ${ }^{111} \mathrm{In}$ no ordered structure is observed by PAC (see Fig. 2, top panel), while X-ray diffraction (XRD) investigations show the signature of a $h c p$ structure. After annealing at $880 \mathrm{~K}$ the PAC data of 

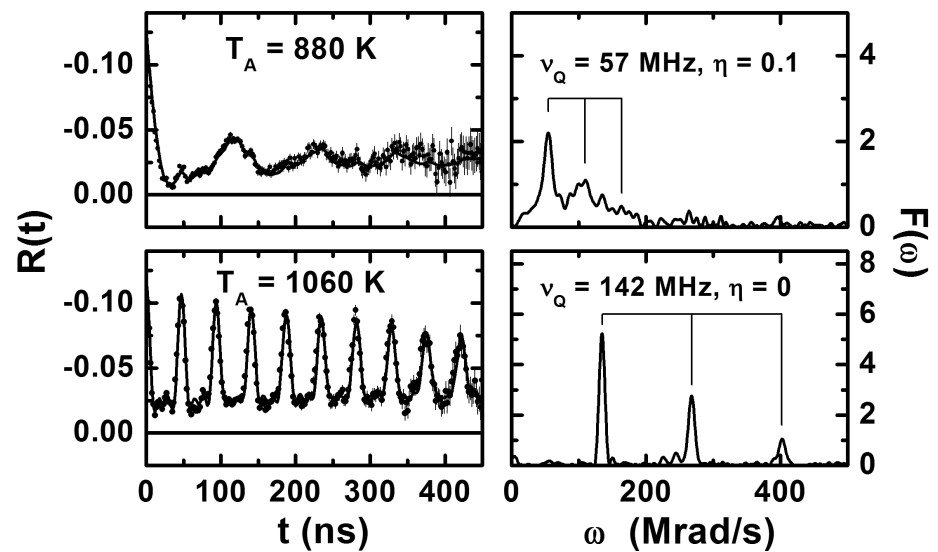

Fig. 1. PAC spectra of a $\mathrm{Ti}_{0.5} \mathrm{Al}_{0.5}$ sample after $4 \mathrm{~h}$ milling time. The alloy was diffused with the ${ }^{111} \mathrm{In}$ probe atoms at $690 \mathrm{~K}$ and subsequently annealed at $880 \mathrm{~K}$ (top panel) and $1060 \mathrm{~K}$ (bottom panel).
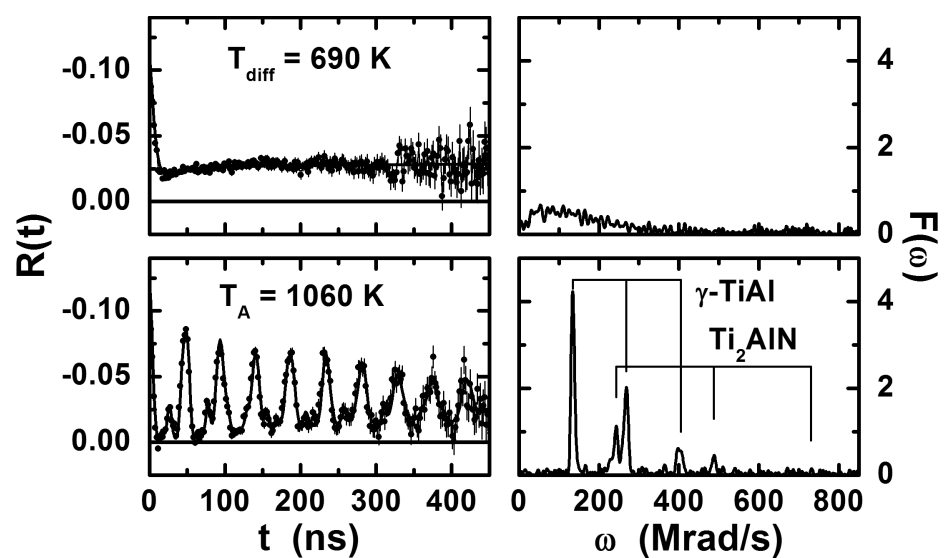

Fig. 2. PAC spectra of a $\mathrm{Ti}_{0.5} \mathrm{Al}_{0.5}$ sample after $13 \mathrm{~h}$ milling time. The alloy was diffused with the ${ }^{111} \mathrm{In}$ probe atoms at $690 \mathrm{~K}$ (top panel) and subsequently annealed at $1060 \mathrm{~K}$ (bottom panel).

the sample milled for $4 \mathrm{~h}$ show an EFG distribution about $57 \mathrm{MHz}$ (Fig. 1, top panel), which is not known from elemental $\mathrm{Ti}$ or $\mathrm{Al}$ samples. At the same time, $\mathrm{XRD}$ investigations just show the $f$ ct structure characteristic of a $\mathrm{Ti}_{0.5} \mathrm{Al}_{0.5}$ alloy. Increasing the annealing temperature to $1060 \mathrm{~K}$, the PAC data show a unique EFG of $142 \mathrm{MHz}$ (Fig. 1, bottom panel), which is known to be caused by the intermetallic compound TiAl [5], while the XRD data remain almost unchanged. Annealing the sample milled for $13 \mathrm{~h}$ at $1060 \mathrm{~K}$, besides the intermetallic compound $\gamma$-TiAl an additional crystalline structure is observed by $\mathrm{PAC}\left(\nu_{Q}=257 \mathrm{MHz}\right.$, see Fig. 2, bottom panel), which on the basis of XRD data is identified as $\mathrm{Ti}_{2} \mathrm{AlN}$. The longer milling time obviously leads to enhanced incorporation of $N$ impurities 
into the $\mathrm{Ti}-\mathrm{Al}$ alloy. More details about the investigations of $\mathrm{Ti}-\mathrm{Al}$ alloys can be found in Ref. [6].

\subsection{Magnetic boundaries in nanocrystalline $\mathrm{Ni}$}

Nanocrystalline Ni was prepared by pulsed electrodeposition (PED) [7]. Short current pulses with a length of $t_{\text {on }}=2 \mathrm{~ms}$ and a current density of $1 \mathrm{~A} / \mathrm{cm}^{2}$ were applied followed by an off-time of $48 \mathrm{~ms}$. The electrolyte used for deposition consisted of $\mathrm{Ni}(\mathrm{II})$ sulfate $(40 \mathrm{~g} / \mathrm{l}), \mathrm{K}$, Na-tartrate $(120 \mathrm{~g} / \mathrm{l})$, and ammonium chloride $(40 \mathrm{~g} / \mathrm{l})$. The average grain size is controlled by the current density, by the temperature of the electrolyte, and by the optional addition of saccharin. For doping of nanocrystalline Ni samples in situ with ${ }^{111} \mathrm{In}$, radioactive ${ }^{111} \mathrm{InCl}_{3}$ was added to the electrolyte. The average grain size of the nanocrystalline Ni samples was determined by XRD.

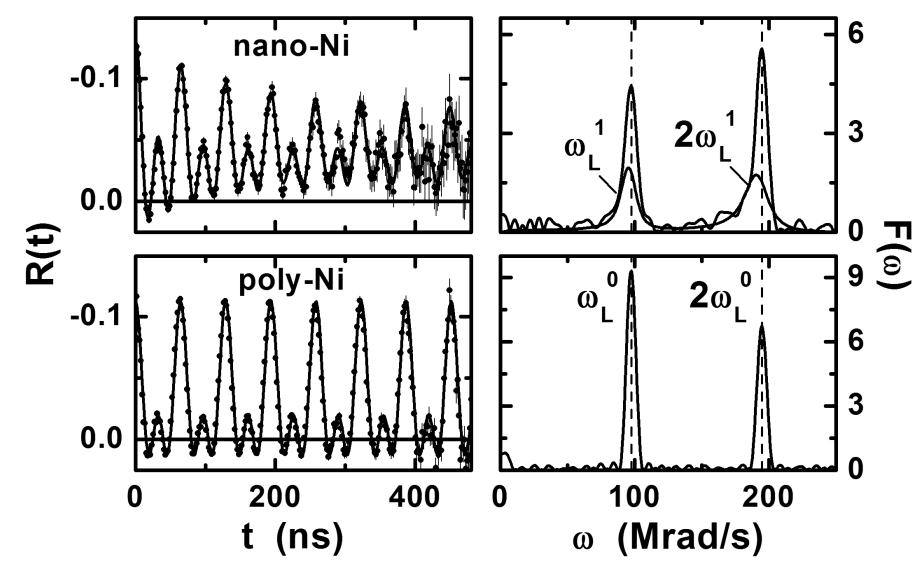

Fig. 3. PAC spectrum of nanocrystalline Ni (top panel) compared to polycrystalline $\mathrm{Ni}$ (bottom panel).

The PAC spectrum plotted in Fig. 3 shows primarily the magnetic dipole interaction of ferromagnetic Ni. A detailed analysis yields that the PAC spectrum consists of two components (top panel). The first one exhibits the sharp Larmor frequency $\omega_{\mathrm{L}}^{0}=97.6 \mathrm{Mrad} / \mathrm{s}$ known from polycrystalline Ni (bottom panels) and caused by the ferromagnetism of $\mathrm{Ni}$. The second component is shifted to a somewhat lower frequency of $\omega_{\mathrm{L}}^{1}=95.8 \mathrm{Mrad} / \mathrm{s}$ and is accompanied by a significant frequency distribution of $\Delta \omega_{\mathrm{L}}^{1}=6 \mathrm{Mrad} / \mathrm{s}$. Due to the preparation conditions it can be assumed that the ${ }^{111} \mathrm{In}$ atoms are homogeneously distributed in the sample. It is suggested, therefore, that the ${ }^{111}$ In atoms exposed to the slightly modified local magnetic field are located near the grain boundaries. Using the relative fractions of about $f_{0}=49 \%$ corresponding to $\omega_{\mathrm{L}}^{0}$ and $f_{1}=37 \%$ corresponding to $\omega_{\mathrm{L}}^{1}$, and the average particle size of about $40 \mathrm{~nm}$, as determined by XRD, it is estimated that the width of the layer corresponding to the modified local magnetic field is 
of the order of 3-4 nm. This so-called magnetic boundary is significantly larger than the crystallographic boundary of about only $1 \mathrm{~nm}$. A detailed description of these experiments can be found in [8].

\subsection{Ordered grain boundary structures in nanocrystalline $\mathrm{Ni}$}

The diffusion of ${ }^{111} \mathrm{In}$ atoms into nanocrystalline $\mathrm{Ni}$ takes place at significantly lower temperatures than into polycrystalline Ni. The PAC data plotted in Fig. 4, obtained after diffusion at $673 \mathrm{~K}$ (top panel), do not show the magnetic interaction known from bulk Ni. Rather, a fraction of about $10 \%$ of the probe atoms shows the signature of an electric quadrupole interaction. Increasing the temperature to $823 \mathrm{~K}$ (middle panel) different signals originating from different well-defined electric field gradients are observed, but still no unique magnetic interaction. After annealing at $1073 \mathrm{~K}$ (bottom panel), both another EFG signal and the signal of the magnetic hyperfine interaction known from bulk $\mathrm{Ni}$ are observed.

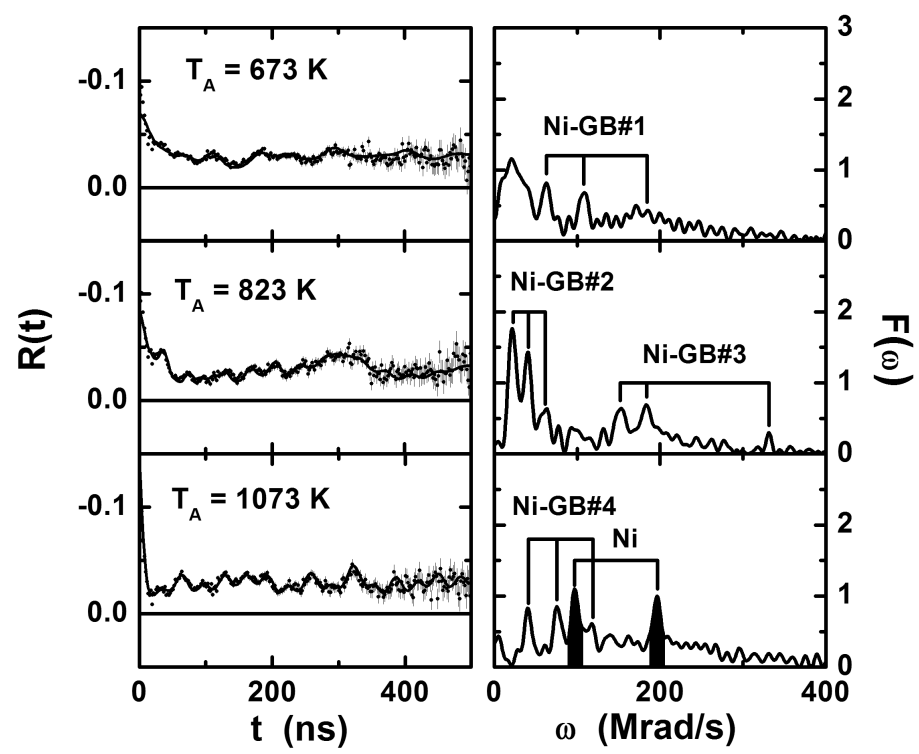

Fig. 4. Ordered grain boundary structures in Ni detected by PAC.

The diffusion of ${ }^{111} \mathrm{In}$ at temperatures below about $1000 \mathrm{~K}$ is determined predominantly by diffusion along grain boundaries [9]. From the PAC data it is concluded that in the grain boundaries obviously no unique magnetic field exists. The presence of sharp EFG signals, however, indicates that there exist ordered structures in the grain boundaries of Ni. Above $1000 \mathrm{~K}$ also the volume diffusion of In becomes important, and a measurable fraction of the ${ }^{111}$ In probe atoms diffuses from the grain boundaries into the crystallites as is shown by the characteristic magnetic hyperfine field. At present, four different ordered grain boundary structures have been detected by PAC [10]. It should be noted that there is no other 
experimental technique able to detect ordered grain boundary structures, with the exception of high resolution electron microscopy, which was successfully applied for the detection of ordered grain boundary structures in $\mathrm{NiO}$ bicrystals [11].

\subsection{Homogeneity of $\mathrm{NiCu}$ alloys}

Nanocrystalline $\mathrm{Ni}-\mathrm{Cu}$ alloys were prepared by PED, using an electrolyte consisting of $\mathrm{NiSO}_{4}, \mathrm{CuSO}_{4}$, and Na-citrate. Optionally, saccharin was added to the electrolyte. For doping the $\mathrm{Ni}-\mathrm{Cu}$ nanoparticles with ${ }^{111} \mathrm{In},{ }^{111} \mathrm{InCl}_{3}$ was added to the electrolyte. Since the deposition of $\mathrm{Cu}$ is much more effective than of $\mathrm{Ni}$, the atomic ratio of $\mathrm{Ni}$ and $\mathrm{Cu}$ in the electrolyte had to be around 13 in order to prepare a $\mathrm{Ni}-\mathrm{Cu}$ alloy with an atomic ratio of 1 . Particle size and homogeneity of the alloy depend on the temperature of the electrolyte, the current density during electro deposition, and on the addition of saccharin to the electrolyte. If no saccharin is added to the electrolyte, the Fourier spectra of the PAC data displayed in Fig. 5 clearly show the presence of Ni precipitates as indicated by the Larmor frequency corresponding to ${ }^{111} \mathrm{In}$ atoms being substitutionally incorporated in bulk

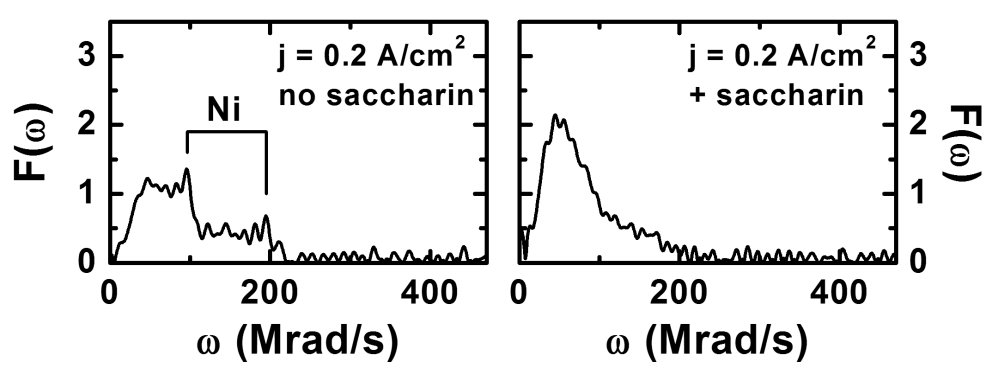

Fig. 5. Ni precipitates observed in a nanocrystalline $\mathrm{NiCu}$ alloy by PAC (left panel). Adding saccharin to the electrolyte (right panel) avoids the precipitation of $\mathrm{Ni}$.

$\mathrm{Ni}$ (left panel) as shown by the absence of the respective Larmor frequency. The $\mathrm{Ni}$ precipitates disappear if saccharin is added during deposition (right panel). The $\mathrm{Ni}$ precipitates cannot be identified on the basis of XRD data, showing the excellent sensitivity of PAC to the formation of these precipitates. More information about the investigations of nanocrystalline $\mathrm{NiCu}$ alloys can be found in Ref. [12].

\subsection{In donors in $\mathrm{ZnO}$}

Nano $\mathrm{ZnO}$ was prepared by the electrochemical deposition under oxidizing conditions (EDOC) method [13]. The electrolysis process uses two Zn electrodes and takes place in a solution of $0.1 \mathrm{M}$ tetrabutylammonium-bromide (TBA-Br) dissolved in 2-propanol. The nanocrystalline $\mathrm{ZnO}$ samples were doped in situ with radioactive ${ }^{111}$ In by diffusing the ${ }^{111}$ In dopants into the sacrificial electrode before electrolysis. After preparation, a hydrothermal treatment at temperatures between $373 \mathrm{~K}$ and $523 \mathrm{~K}$ was applied to the $\mathrm{ZnO}$ nanocrystals. More details about the EDOC method and the hydrothermal treatment are given in [14]. The 
incorporation of In atoms on substitutional lattice sites is determined by PAC if the EFG due to the hexagonal lattice structure of $\mathrm{ZnO}$, which is known from bulk $\mathrm{ZnO}$ [15], is detected. The $\mathrm{ZnO}$ nanocrystals were investigated in parallel by transmission electron microscopy and XRD in order to determine shape, size, size distribution, and microstrain of the particles, and by absorption and photoluminescence spectroscopy in order to determine their optical properties.

Comprehensive investigations show that after hydrothermal annealing at $473 \mathrm{~K}$ the average size of the spherical particles is increased from $4 \mathrm{~nm}$ to $11 \mathrm{~nm}$; at the same time the microstrain present after deposition disappears. Furthermore, the absorption power and the excitonic emission after laser excitation are increased. The PAC investigations displayed in Fig. 6 show no detectable fraction directly after deposition (top panel) but after hydrothermal treatment at $473 \mathrm{~K}$ about $20 \%$ of the ${ }^{111} \mathrm{In}$ atoms were incorporated on substitutional Zn sites (bottom panel). Increasing the annealing temperature to $523 \mathrm{~K}$ the optical properties

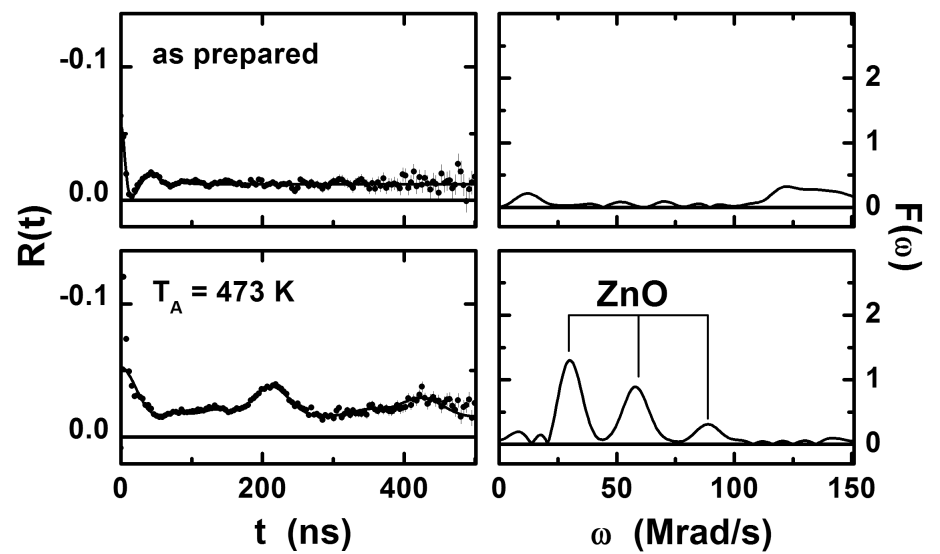

Fig. 6. PAC spectra of nanocrystalline $\mathrm{ZnO}$ after deposition (top panels) and after subsequent hydrothermal annealing at $473 \mathrm{~K}$ (bottom panels). The modulation caused by an EFG shows the incorporation of ${ }^{111}$ In atoms on substitutional $\mathrm{Zn}$ sites.

changed only slightly and the fraction of ${ }^{111}$ In atoms on substitutional Zn sites was slightly increased. The particles, however, have grown significantly into about $200 \mathrm{~nm}$ long rod-shaped crystals. Thus, the temperature of $473 \mathrm{~K}$ was identified as being an optimum for producing $\mathrm{ZnO}$ nanocrystals of good crystalline quality doped with In atoms on substitutional lattice sites. The full description of the experimental analysis of nanocrystalline $\mathrm{ZnO}$ is found in Ref. [14].

\section{Summary}

The investigations of nanocrystalline materials by PAC yield valuable, in part unique, information about crystallites and grain boundaries. It turned out that in some cases the sensitivity of PAC to phenomena, like ordered grain boundary 
structures or precipitates in alloys, is much higher than that of other experimental techniques. By PAC investigations the donor-like incorporation of In atoms into $\mathrm{ZnO}$ nanocrystals was shown.

\section{Acknowledgments}

The experimental help of St. Lauer, Z. Guan, Th. Agne, C.E. Krill, and J. Schmauch, the technical support of prof. R. Birringer, prof. R. Hempelmann and H. Natter, and the financial support of the DFG in the framework of the SFB 277 is gratefully acknowledged.

\section{References}

[1] H. Gleiter, Prog. Mater. Sci. 33, 223 (1989).

[2] G. Schatz, A. Weidinger, Nuclear Condensed Matter Physics, Wiley, Chichester (England) 1995, p. 63.

[3] Th. Wichert, E. Recknagel in: Topics in Current Physics, Ed. U. Gonser, Vol. 40, Berlin 1986, p. 317.

[4] Th. Wichert, in: Identification of Defects in Semiconductors, Ed. M. Stavola, Academic Press, London 1999, p. 297.

[5] J. Fan, G.S. Collins, Hyp. Int. 79, 745 (1993).

[6] St. Lauer, Z. Guan, H. Wolf, Th. Wichert, J. Mater. Res. 17, 2130 (2002).

[7] H. Natter, M. Schmelzer, R. Hempelmann, J. Mater. Res. 13, 1186 (1998).

[8] St. Lauer, Z. Guan, H. Wolf, H. Natter, M. Schmelzer, R. Hempelmann, Th. Wichert, Nanostruct. Mater. 12, 955 (1999).

[9] I. Kaur, W. Gust, L. Kozma, Handbook of Grain and Interphase Boundary Diffusion Data, Ziegler Press, Stuttgart 1989, p. 1003.

[10] Z. Guan, H. Wolf, X. Li, Th. Wichert, Hyp. Int. 136/137, 281 (2001); Z. Guan, H. Wolf, X. Li, Th. Agne, Th. Wichert, in preparation for publication.

[11] K.L. Merkle, J.F. Reddy, C.L. Willy, D.J. Smith, Phys. Rev. Lett. 59, 2887 (1987).

[12] H. Wolf, Z. Guan, St. Lauer, H. Natter, M. Schmelzer, R. Hempelmann, Th. Wichert, J. Metast. Nanocryst. Mater. 8, 847 (2000).

[13] A. Dierstein, H. Natter, F. Meyer, H.-O. Stephan, Ch. Kropf, R. Hempelmann, Scr. Mater. 44, 2209 (2001).

[14] Th. Agne, Z. Guan, R. Hempelmann, X. Li, H. Natter, H. Wolf, Th. Wichert, Appl. Phys. Lett. 83, 1204 (2003).

[15] H. Wolf, S. Deubler, D. Forkel, H. Foettinger, M. Iwatschenko-Borho, F. Meyer, M. Renn, W. Witthuhn, R. Helbig, Mater. Sci. Forum 10-12, 863 (1986). 